\title{
Spectral Analysis of the Light Scattered by a Ternary Mixture
}

\author{
BY HENDRIK N. W. LEKKERKERKER \\ Fakulteit van de Wetenschappen, Vrije Universiteit Brussels, \\ 1050 Brussels, Belgium \\ AND \\ WILLIAM G. LAIDLAW* \\ Department of Chemistry, The University of Calgary, \\ Calgary, Alberta, Canada T2N 1N4
}

Received 25th September, 1973

\begin{abstract}
The normal mode analysis of the light scattered by a ternary mixture is presented. The treatment is based upon a method, previously formulated by the authors, which is valid under conditions for which the sound modes are decoupled from the modes associated with heat and mass diffusion. The expressions obtained for both the lorentzian and non-lorentzian contributions to the Brillouin peaks are straightforward extensions of those obtained for the one and two component cases. The damping and strength factors of the three lorentzian contributions to the Rayleigh peak are rather complicated functions of the transport coefficients and thermodynamic derivatives. As illustrative examples the situations where, $(a)$ one species and $(b)$ two species in the ternary mixture are present in low concentration, are considered in some detail.
\end{abstract}

There has been considerable activity, both experimentally and theoretically in the study of the spectral distribution of scattered light. ${ }^{1}$ In the usual macroscopic calculation of the light scattering spectrum one utilizes the Onsager assumption concerning the regression of fluctuations to obtain the spectral densities in terms of which the spectral intensity of the light can be expressed. To facilitate the analysis of the spectrum a normal mode decomposition is frequently undertaken and to carry out this decomposition one first has to obtain the eigenvalues of the hydrodynamic matrix and subsequently perform either a partial fraction decomposition or, as will be done here, obtain the required expression in terms of the eigenvectors of the hydrodynamic matrix.

General procedures for obtaining the normal mode decomposition for multicomponent fluids have been presented by Blum ${ }^{2}$ and by Jordan and Jordan. ${ }^{3}$ The former utilized a perturbation expansion in the scattering vector $\boldsymbol{k}$ while the latter featured the use of normalized statistically independent variables and the selection of the appropriately normalized pressure fluctuation as one of these variables. The present authors have extended ${ }^{4}$ the work of Jordan and Jordan and systematized a procedure for obtaining satisfactory approximations to the eigenvalues and eigenvectors from a partitioned form of the hydrodynamic matrix. As an illustration of this procedure we considered ${ }^{4}$ the spectral distribution of light scattered from a binary mixture, a system already examined by Martin, ${ }^{5}$ Jordan and Jordan, ${ }^{3}$ Blum, ${ }^{2}$ Mountain and Deutch ${ }^{6}$ and Cohen et al. ${ }^{7}$

The use of normal mode expressions in the analysis of the experimental light scattering spectrum of a binary mixture facilitates obtaining ${ }^{8}$ transport and thermodynamic properties of such systems. Similarly the use of light scattering spectroscopy to study multicomponent fluids could be furthered by the use of a normal mode 
decomposition of the spectrum. Ternary mixtures have been studied in terms of a third species dissolved in a binary mixture in particular by Goldburg and Pusey ${ }^{9}$ near the critical point and by Ford et al. ${ }^{10}$ for the noncritical region. Only the latter situation is of concern to us and in this paper we obtain, under conditions for which the sound modes are decoupled from the modes associated with heat and mass diffusion, the appropriate normal mode expressions for a ternary mixture.

The complex eigenvalues are obtained as the roots of a second degree polynomial and are straightforward extensions of those obtained for a two component system. The real eigenvalues are obtained as the zeros of a third degree polynomial. Rather than considering the unwieldy expressions which result for the real eigenvalues, a perturbation treatment, in which the thermal diffusion terms are taken to be small, is employed. The results obtained for the non-propagating normal modes are illustrated by considering, in some detail, the two special cases where: $(a)$ one component and, $(b)$ two components in the ternary mixture are present in low concentration.

\section{SPECTRUM OF SCATTERED LIGHT}

We refer to our earlier papers ${ }^{4,11}$ for the development of the argument which allows one to write down the expression for the spectral distribution of the light scattered by a multicomponent fluid as

$$
I(k, \omega)=C \sum_{l, m}\left(\frac{\partial \varepsilon}{\partial B_{l}}\right)\left(\frac{\partial \varepsilon}{\partial B_{m}}\right) \frac{1}{\pi} \operatorname{Re} \sum_{f} \frac{V_{l f} V_{m f}}{\lambda_{f}+\mathrm{i} \omega} .
$$

Here $k$ is the magnitude of the scattering vector, $\omega$ is the shift in frequency of the scattered light and $C$ contains experimental parameters and physical constants which will not concern us here. The quantities $\left(\partial \varepsilon / \partial B_{l}\right)$ are the derivatives of the optical dielectric constant appropriate to the fluctuations $\beta_{l}$, the spatial fourier components of which satisfy the relations $\left\langle\beta_{l}(k) \beta_{m}^{*}(k)\right\rangle=\delta_{l m}$. The fluctuations in the optical dielectric constant are determined by fluctuations in the thermodynamic state variables and therefore the summation over $l$ and $m$ in eqn (1) extends only over the even variables of the set $\left\{\beta_{l}\right\}$ since these represent the fluctuations in the thermodynamic variables of the system. The $\lambda_{f}$ and the $\mathrm{V}_{t f}$ represent eigenvalues and eigenvector elements respectively of the hydrodynamic matrix $\boldsymbol{K}(k)$ which results upon expressing a spatially fourier transformed set of hydrodynamic equations in terms of the variables $\beta_{l}(\boldsymbol{k}, t)$.

\section{THE SELECTION OF VARIABLES AND THE HYDRODYNAMIC MATRIX}

The hydrodynamic equations for a three component fluid can be readily obtained using the methods of irreversible thermodynamics ${ }^{12}$ and in linearized form can be written as:

$$
\begin{aligned}
\frac{\partial \rho}{\partial t} & =-\rho_{0} \operatorname{div} v \\
\rho_{0} \frac{\partial}{\partial t} \operatorname{div} v & =-\nabla^{2} p+\left(\frac{4}{3} \eta+\zeta\right) \nabla^{2} \operatorname{div} v \\
\rho_{0} \frac{\partial c_{i}}{\partial t} & =-\operatorname{div} \boldsymbol{J}_{i} \quad(i=1,2) \\
\rho_{0} T_{0} \frac{\partial s}{\partial t} & =-\operatorname{div} \boldsymbol{J}_{q, \mathrm{red}}-T_{0} \sum_{i=1,2}\left(\frac{\partial s}{\partial c_{i}}\right)_{p T c_{j \neq i}} \operatorname{div} \boldsymbol{J}_{i} .
\end{aligned}
$$


Here $\boldsymbol{J}_{1}$ and $\boldsymbol{J}_{2}$ are the diffusion flows of components 1 and 2 respectively and $\boldsymbol{J}_{q, \text { red }}$ is the reduced heat flow. ${ }^{13}$ These fluxes are defined by:

$$
\begin{aligned}
& \boldsymbol{J}_{i}=-\frac{\rho_{0} D_{i}^{\mathrm{T}}}{T_{0}} \operatorname{grad} T-\rho_{0} \sum_{j=1,2} D_{i j} \operatorname{grad} c_{j}- \\
&\left.\frac{\rho_{0}}{\mid \boldsymbol{G}}\right|_{m, j=1,2} D_{i m}\left(\boldsymbol{G}^{-1}\right)_{m j}\left(\frac{\partial \hat{\mu}_{j}}{\partial p}\right)_{T c_{1} c_{2}} \operatorname{grad} p \quad(i=1,2)
\end{aligned}
$$

$\boldsymbol{J}_{q, \mathrm{red}}=-\lambda \operatorname{grad} T-\rho_{0} \sum_{l, m=1,2} D_{m}^{\mathrm{T}} G_{m l} \operatorname{grad} c_{l}-\rho_{0} \sum_{m=1,2} D_{m}^{T}\left(\frac{\partial \hat{\mu}_{m}}{\partial p}\right)_{T c_{1} c_{2}} \operatorname{grad} p$.

The thermodynamic variables appearing in eqn (2) to (7) have the following meaning: $\rho$ is the mass density, $T$ is the temperature, $p$ is the pressure, $s$ is the specific entropy, $c_{1}$ and $c_{2}$ are the mass fractions of components 1 and 2 respectively, $\hat{\mu}_{i}=\mu_{i}-\mu_{3}$ $(i=1,2)$ where $\mu_{i}$ is the chemical potential of component $i$ per unit mass, $\hat{\mu}_{i j} \equiv$ $\left(\partial \hat{\mu}_{i} / \partial c_{j}\right)_{p T c_{l \neq j}}$ and the elements of the symmetric matrix $\boldsymbol{G}$ are defined as $G_{i j}=\hat{\mu}_{i j}$ $(i, j=1,2)$. The subscript 0 implies the average value for that variable. Except for cases where it could give rise to confusion we will henceforth omit the subscript 0 . The transport coefficients that appear in eqn (3), (6) and (7) are the shear viscosity $\eta$, the bulk viscosity $\zeta$, the diffusion coefficients $D_{i j}$, the thermal diffusion coefficients $D_{i}^{T}$ and the heat conductivity coefficient $\lambda$. The diffusion coefficients $D_{i j}$ are not all independent since, by virtue of the Onsager symmetry relations, one has the requirement ${ }^{14}$ :

$$
-D_{11} \hat{\mu}_{12}+D_{12} \hat{\mu}_{11}=D_{21} \hat{\mu}_{22}-D_{22} \hat{\mu}_{12} .
$$

The procedure for normal mode analysis, alluded to at the outset, requires that the hydrodynamic equations be recast to give these equations in terms of normalized statistically independent fluctuations $\beta_{l}(\boldsymbol{k}, t)$ with the normalized pressure fluctuation as one of that set. We choose, here, as the statistically independent fluctuations the set $\delta s_{\text {red }}, \delta c_{1, \text { red }}, \delta c_{2}, \delta p$ and div $\boldsymbol{v}$ where

$$
\begin{gathered}
\delta s_{\text {red }}=\delta s-\sum_{i=1,2}\left(\frac{\partial s}{\partial c_{i}}\right)_{p T c_{l \neq i}} \delta c_{\mathrm{i}} \\
\delta c_{1, \mathrm{red}}=\delta c_{1}-\left(\frac{\partial c_{1}}{\partial c_{2}}\right)_{p T \hat{\mu}_{1}} \delta c_{2} .
\end{gathered}
$$

The statistical independence of $\operatorname{div} v$ from the remaining fluctuation variables follows directly from considerations of microscopic reversibility ${ }^{15}$ for $\operatorname{div} v$ is odd and the other variables are even under time reversal. The statistical independence of the remaining fluctuation variables can be established using thermodynamic fluctuation theory. ${ }^{16}$ The physical interpretation of such reduced variables may be appreciated through the appropriate equation of motion, for example, for $\delta s_{\text {red }}$ one can easily establish that

$$
\rho T \frac{\partial \delta s_{\mathrm{red}}}{\partial t}=-\operatorname{div} \boldsymbol{J}_{q, \mathrm{red}} .
$$

In the limit of small $\boldsymbol{k}$, the normalized spatial fourier components of the above set of variables are given by ${ }^{16}$

$$
\begin{aligned}
& \beta_{1}(\boldsymbol{k}, t)=\left[k_{\mathrm{B}} T V C_{p} / \rho T\right]^{-\frac{1}{2}} \delta s_{\mathrm{red}}(\boldsymbol{k}, t) \\
& \beta_{2}(\boldsymbol{k}, t)=\left[k_{\mathrm{B}} T V / \rho \hat{\mu}_{11}\right]^{-\frac{1}{2}} \delta c_{1, \mathrm{red}}(\boldsymbol{k}, t)
\end{aligned}
$$




$$
\begin{aligned}
& \beta_{3}(\boldsymbol{k}, t)=\left[k_{\mathrm{B}} T V / \rho\left(\frac{\partial \hat{\mu}_{2}}{\partial c_{2}}\right)_{p T \hat{\mu}_{1}}\right]^{-\frac{1}{2}} \delta c_{2}(\boldsymbol{k}, t) \\
& \beta_{4}(\boldsymbol{k}, t)=\left[k_{\mathrm{B}} T V / \chi_{\mathrm{s}}\right]^{-\frac{1}{2}} \delta p(\boldsymbol{k}, t) \\
& \beta_{5}(\boldsymbol{k}, t)=\left[k_{\mathrm{B}} T V k^{2} / \rho\right]^{-\frac{1}{2}}(\operatorname{div} \boldsymbol{v})(\boldsymbol{k}, t) .
\end{aligned}
$$

In eqn (11) $k_{\mathrm{B}}$ is Boltzmann's constant, $V$ is the volume in which the fluctuations are considered, $C_{p}$ is the specific heat at constant pressure and $\chi_{\mathrm{s}}$ is the adiabatic compressibility. Rewriting the hydrodynamic eqn (2) to (5) in terms of the variables $\delta s_{\text {red }}, \delta c_{1, \text { red }}, \delta c_{2}, \delta p$ and div $v$, spatially fourier transforming the resulting equations and introducing the variables $\beta_{l}(k, t)$ defined by eqn (11) one obtains for the hydrodynamic equations

$$
\frac{\partial \beta}{\partial t}(\boldsymbol{k}, t)=-\boldsymbol{K}(k) \beta(\boldsymbol{k}, t) .
$$

Taking into account the symmetry relation ${ }^{11} \boldsymbol{K}^{\mathrm{T}}=\boldsymbol{E} \boldsymbol{K} \boldsymbol{E}$ where the so called signature matrix $\boldsymbol{E}$ is, in the present case, given by

$$
E=\operatorname{diag}\{1,1,1,1,-1\}
$$

and considering the fact that $\operatorname{div} v$ is coupled only to div $v$ and to the pressure, it follows that $\boldsymbol{K}(k)$ has the structure displayed in eqn (13)

$$
\boldsymbol{K}=\left[\begin{array}{ccc:cc} 
& & K_{14} & 0 \\
& & & K_{24} & 0 \\
K_{34} & 0 \\
\hdashline K_{14} & K_{24} & K_{34} & K^{\prime \prime} \\
0 & 0 & 0 &
\end{array}\right]
$$

In eqn (13) $\boldsymbol{K}^{1}$ is the $3 \times 3$ symmetric matrix given by eqn (14), $\boldsymbol{K}^{11}$ is the $2 \times 2$ antisymmetric matrix displayed in eqn (15) and the remaining non-zero elements of $\boldsymbol{K}$, namely $K_{j 4}=K_{4 j}(j=1,2,3)$ are given by eqn (16) to (18)

$$
\boldsymbol{K}^{1}=\left[\begin{array}{lll}
D_{\mathrm{T}} k^{2} & {\left[D_{1}^{T}-\left(\frac{\partial c_{1}}{\partial c_{2}}\right)_{p T \hat{\mu}_{1}} D_{2}^{T}\right]\left[\frac{\hat{\mu}_{11}}{C_{p} T}\right]^{\frac{1}{2}} k^{2}} & D_{2}^{T}\left[\frac{\left(\partial \hat{\mu}_{2} / \partial c_{2}\right)_{p T \hat{\mu}_{1}}}{C_{p} T}\right]^{\frac{1}{2}} k^{2} \\
K_{12} & {\left[D_{11}-\left(\frac{\partial c_{1}}{\partial c_{2}}\right)_{p T \hat{\mu}_{1}} D_{21}\right] k^{2}} & D_{21}\left[\frac{\left(\partial \hat{\mu}_{2} / \partial c_{2}\right)_{p T \hat{\mu}_{1}}}{\hat{\mu}_{11}}\right]^{\frac{1}{2}} k^{2} \\
K_{13} & K_{23} & {\left[\left(\frac{\partial c_{1}}{\partial c_{2}}\right)_{p T \hat{\mu}_{1}} D_{21}+D_{22}\right] k^{2}}
\end{array}\right] .
$$

Here $D_{\mathrm{T}}=\lambda / \rho C_{p}$ and using eqn (8) we have expressed $D_{12}$ in terms of $D_{11}, D_{22}$ and $D_{21}$.

$$
\boldsymbol{K}^{\mathbf{I I}}=\left[\begin{array}{cc}
\left\{D_{T}(\gamma-1)+\frac{2 c_{0}^{2} \alpha \rho}{C_{p}} \sum_{l=1,2} D_{l}^{T} \hat{v}_{l}+c_{0}^{2} \rho^{2} \hat{\boldsymbol{v}}^{T} \boldsymbol{D} \boldsymbol{G}^{-1} \hat{\boldsymbol{v}}\right\} k^{2} & k c_{0} \\
-k c_{0} & \frac{\left(\frac{4}{3} \eta+\zeta\right)}{\rho} k^{2}
\end{array}\right] .
$$

Here $\alpha$ is the thermal expansion coefficient, $\gamma$ is the ratio of the specific heats at constant pressure and at constant volume, $c_{0}=\left(\rho \chi_{\mathrm{s}}\right)^{-\frac{1}{2}}$ is the low frequency sound 
velocity, $\hat{v}_{l}=v_{l}-v_{3}(l=1,2)$ where $v_{l}$ is the partial specific volume of component $l$ and $\hat{v}$ is a column vector with elements $\hat{v}_{1}$ and $\hat{v}_{2}$.

$$
\begin{aligned}
& K_{14}=\left\{\rho T\left(\frac{\partial v}{\partial T}\right)_{p c_{1} c_{2}} D_{T}+\rho \sum_{l} D_{l}^{T} \hat{v}_{l}\right\}\left(\rho T C_{p} \chi_{s}\right)^{-\frac{1}{2}} k^{2} \\
& K_{24}=\left\{\frac{\alpha}{C_{p}}\left(\sum_{l=1,2} D_{l}^{T} \hat{\mu}_{1 l}\right)+\rho\left(\sum_{l=1,2} D_{l 1} \hat{v}_{l}\right)\right\}\left(\rho \hat{\mu}_{11} \chi_{\mathrm{s}}\right)^{-\frac{1}{2}} k^{2} \\
& K_{34}=\left\{\frac{\alpha}{C_{p}}\left(\frac{\partial \hat{\mu}_{2}}{\partial c_{2}}\right)_{p T \hat{\mu}_{1}} D_{2}^{T}+\rho\left(\frac{\hat{\mu}_{22} \hat{v}_{1}-\hat{\mu}_{12} \hat{v}_{2}}{\hat{\mu}_{11}}\right) D_{21}+\right. \\
& \left.\rho\left(\frac{\hat{\mu}_{11} \hat{v}_{2}-\hat{\mu}_{12} \hat{v}_{1}}{\hat{\mu}_{11}}\right) D_{22}\right\}\left(\rho\left(\frac{\partial \hat{u}_{2}}{\partial c_{2}}\right)_{p T \hat{\mu}_{1}} \chi_{\mathrm{s}}\right)^{-\frac{1}{2}} k^{2} .
\end{aligned}
$$

THE HYDRODYNAMIC NORMAL MODES

Again referring to our earlier work ${ }^{4}$ for the details it can be shown that in the case that the transport terms $D_{\mathrm{T}} k^{2}, D_{i j} k^{2}, D_{i}^{T} k^{2}$ and $\left(\left(\frac{4}{3} \eta+\zeta\right) / \rho\right) k^{2}$ are much smaller than the sound frequency $k c_{0}$, the eigenvalues and eigenvectors of the hydrodynamic matrix can be obtained as follows.

(i) The roots of $\boldsymbol{K}^{\mathbf{1}}, \lambda_{1,2,3}^{\mathrm{I}}$, are a satisfactory (see below) approximation to the real eigenvalues $\lambda_{1,2,3}$ of $\boldsymbol{K}$ and similarly the roots of $\boldsymbol{K}^{\mathrm{II}},\left(\lambda_{1}^{\mathrm{II}}\right)^{*}=\lambda_{2}^{\text {II }}$ are a satisfactory approximation to the complex eigenvalues $\lambda_{4}^{*}=\lambda_{5}$ of $\boldsymbol{K}$.

(ii) The eigenvector elements of $\boldsymbol{K}$, required in eqn (1) can, to the same degree of accuracy, be represented by

$$
\boldsymbol{V}=\left[\begin{array}{ccc|ll} 
& & & V_{14}^{(1)} & V_{14}^{(1) *} \\
& & & V_{24}^{(1)} & V_{24}^{(1) *} \\
& & & V_{34}^{(1)} & V_{34}^{(1) *} \\
\hdashline 0 & 0 & 0 & V_{11}^{\mathrm{II}} & V_{11}^{\mathrm{II} *} \\
x & x & x & x & x
\end{array}\right]
$$

$\boldsymbol{V}^{\mathbf{I}}$ and $\boldsymbol{V}^{\text {II }}$ are the diagonalizing matrices of $\boldsymbol{K}^{\mathrm{I}}$ and $\boldsymbol{K}^{\mathrm{II}}$ respectively and the elements $V_{j 4}^{(1)}(j=1,2,3)$ can be written down directly (see ref. (4) eqn (12)). The first corrections ${ }^{4}$ to the above eigenvalues and eigenvector elements are of relative order $\left(a / k c_{0}\right)^{2}$ where $a$ refers generically to the transport terms in the hydrodynamic matrix. Since for normal fluids $a \ll k c_{0}$ the first corrections are small and the formulations (i) and (ii) above for the eigenvalues and eigenvectors are satisfactory.

Drawing on the above results we now turn to the calculation of the hydrodynamic normal modes. The damping factors of the non-propagating normal modes, i.e. the real eigenvalues of $\boldsymbol{K}$, can then be written as

$$
\lambda_{m+1}=\frac{q_{1}}{3}+\frac{1}{2}\left[q_{1}^{2}-3 q_{2}\right]^{\frac{1}{2}} \cos \left(\phi+2 \frac{\pi m}{3}\right) \quad(m=0,1,2)
$$

Here $q_{1}$ is the trace of $\boldsymbol{K}^{\mathbf{I}}, q_{2}$ is the sum of the principal minors of order 2 of $\boldsymbol{K}^{\mathbf{I}}$ and $q_{3}$ is the determinant of $\boldsymbol{K}^{\mathbf{I}}$ and

$$
\cos \phi=\frac{\left(2 q_{1}^{3}-9 q_{1} q_{2}+27 q_{3}\right)}{2\left(q_{1}^{2}-3 q_{2}\right)^{\frac{3}{2}}}
$$

In turn one can, after considerable algebra, obtain the corresponding eigenvector elements. 
In liquid systems the terms due to thermal diffusion are rather small and approximate analytic expressions for the eigenvalues and eigenvectors of $\boldsymbol{K}^{\mathbf{1}}$ can be obtained using perturbation theory. One writes

$$
K^{1}=K^{1(0)}+K^{1(1)}
$$

where $\boldsymbol{K}^{\mathrm{I}(0)}$ contains all elements of $\boldsymbol{K}^{1}$, except the ones that are treated as perturbations, i.e., $K_{12}\left(K_{21}\right)$ and $K_{13}\left(K_{31}\right)$. The eigenvalues of $K^{1(0)}$ are

$$
\begin{aligned}
& \lambda_{1}^{\mathrm{I}(0)}=D_{\mathrm{T}} k^{2} \\
& \lambda_{2,3}^{\mathrm{I}(0)}=\frac{1}{2}\left[\left(D_{11}+D_{22}\right) \pm\left\{\left(D_{11}-D_{22}-2\left(\frac{\partial c_{1}}{\partial c_{2}}\right)_{p T \hat{\mu}_{1}} D_{21}\right)^{2}+\right.\right. \\
& \left.\left.\left.4 D_{21}^{2} \frac{\left(\partial \hat{\mu}_{2} / \partial c_{2}\right)_{p T \hat{\mu}_{1}}}{\hat{\mu}_{11}}\right\}\right]^{\frac{1}{2}}\right] k^{2}
\end{aligned}
$$

and the orthogonal diagonalizing matrix of $\boldsymbol{K}^{\mathbf{I}(0)}$ can be written as

$$
V^{\mathbf{I}(0)}=\left[\begin{array}{rrr}
1 & 0 & 0 \\
0 & p & -q \\
0 & q & p
\end{array}\right]
$$

where

$$
\begin{aligned}
& p=\left[\frac{\lambda_{2}^{I(0)}-K_{33}}{\lambda_{2}^{(0)}-\lambda_{3}^{I(0)}}\right]^{\frac{1}{2}} \\
& q=\operatorname{sign}\left(K_{23}\right)\left[\frac{\lambda_{3}^{I(0)}-K_{33}}{\lambda_{3}^{I(0)}-\lambda_{2}^{(0)}}\right]^{\frac{1}{2}} .
\end{aligned}
$$

Using standard expressions ${ }^{17}$ of perturbation theory for symmetric matrices one obtains, retaining the first non-vanishing perturbation terms, the following expressions for the eigenvalues and eigenvectors of the matrix $K^{1}$

$$
\begin{gathered}
\lambda_{1}=\lambda_{1}^{I(0)}+\frac{\left(K_{12}^{\prime}\right)^{2}}{\lambda_{1}^{I(0)}-\lambda_{2}^{I(0)}}+\frac{\left(K_{13}^{\prime}\right)^{2}}{\lambda_{1}^{I(0)}-\lambda_{3}^{I(0)}} \\
\lambda_{i}=\lambda_{i}^{I(0)}-\frac{\left(K_{1 i}^{\prime}\right)^{2}}{\lambda_{1}^{I(0)}-\lambda_{i}^{(0)}} \quad(i=2,3) \\
0 \quad V^{I(0)}+\left[\begin{array}{ccc}
\frac{-K_{12}^{\prime}}{\lambda_{1}^{I(0)}-\lambda_{2}^{(0)}} & \frac{-K_{13}^{\prime}}{\lambda_{1}^{I(0)}-\lambda_{3}^{I(0)}} \\
\frac{K_{12}^{\prime} p}{\lambda_{1}^{I(0)}-\lambda_{2}^{I(0)}}+\frac{-K_{13}^{\prime} q}{\lambda_{1}^{I(0)}-\lambda_{3}^{I(0)}} & 0 & 0 \\
\frac{K_{12}^{\prime} q}{\lambda_{1}^{I(0)}-\lambda_{2}^{I(0)}}+\frac{K_{13}^{\prime} p}{\lambda_{1}^{I(0)}-\lambda_{3}^{I(0)}} & 0 & 0
\end{array}\right] .
\end{gathered}
$$

Here $K_{12}^{\prime}$ and $K_{13}^{\prime}$ are elements of the matrix $\left(\boldsymbol{V}^{\mathrm{I}(0)}\right)^{\mathrm{T}} \boldsymbol{K}^{\mathrm{I}(1)} \boldsymbol{V}^{\mathrm{I}(0)}$

$$
\begin{aligned}
& K_{12}^{\prime}=K_{12} p+K_{13} q \\
& K_{13}^{\prime}=-K_{12} q+K_{13} p .
\end{aligned}
$$


The propagating modes are readily obtained irrespective of the number of components since $\boldsymbol{K}^{\mathrm{II}}$ remains a $2 \times 2$ matrix. The complex eigenvalues of the hydrodynamic matrix are given by

where

$$
\lambda_{4,5}=\Gamma \pm \mathrm{i} k c_{0}
$$

$$
\Gamma=\left(K_{44}+K_{55}\right) / 2=\Gamma_{\text {heat conduction }}+\Gamma_{\text {diffusion }}+\Gamma_{\text {thermal diffusion }}+\Gamma_{\text {viscosity }}
$$

A similar result for this damping factor has been obtained by Blum. ${ }^{2}$ The elements of the corresponding eigenvectors needed for eqn (1) are given by

$$
\begin{aligned}
& V_{44}=V_{45}^{*}=\frac{1}{\sqrt{ } 2}\left[1-\frac{\mathrm{i}\left(K_{44}-K_{55}\right)}{4 k c_{0}}\right] \\
& V_{j 4}^{(1)}=V_{j 5}^{(1) *}=-\frac{\mathrm{i} K_{j 4}}{k c_{0} \sqrt{2}} \quad(j=1,2,3)
\end{aligned}
$$

\section{NORMAL MODE CONTRIBUTIONS TO THE LIGHT SCATTERING SPECTRUM}

The spectral intensity distribution of scattered light given by eqn (1) can be split into two parts

$$
I(k, \omega)=I^{\mathrm{R}}(k, \omega)+I^{\mathrm{B}}(k, \omega) .
$$

The first term, which is associated with the non-propagating normal modes, is centred at $\omega=0$ and is referred to as the Rayleigh peak. The second term which is associated with the propagating normal modes consists of lines centred respectively at $\omega= \pm k c_{0}$ and are commonly referred to as the Stokes and anti-Stokes Brillouin peaks.

In the present case the Rayleigh peak can be written as the sum of three lorentzians

$$
I^{\mathrm{R}}(k, \omega)=C \frac{1}{\pi} \sum_{f=1}^{3} Z_{f} \frac{\lambda_{f}}{\lambda_{f}^{2}+\omega^{2}} .
$$

Starting from eqn (1) and using the approximate eigenvector elements given by eqn (25), the strength factors $Z_{f}(f=1,2,3)$ can be written down as

$$
\begin{aligned}
& Z_{1}=\left(\frac{\partial \varepsilon}{\partial B_{1}}\right)^{2}+A+B \\
& Z_{2}=\left\{\left(\frac{\partial \varepsilon}{\partial B_{2}}\right) p+\left(\frac{\partial \varepsilon}{\partial B_{3}}\right) q\right\}^{2}-A \\
& Z_{3}=\left\{-\left(\frac{\partial \varepsilon}{\partial B_{2}}\right) q+\left(\frac{\partial \varepsilon}{\partial B_{3}}\right) p\right\}^{2}-B .
\end{aligned}
$$

Here

$$
\begin{aligned}
& A=2\left(\frac{\partial \varepsilon}{\partial B_{1}}\right) \frac{K_{12}^{\prime}}{\lambda_{1}^{I(0)}-\lambda_{2}^{I(0)}}\left\{\left(\frac{\partial \varepsilon}{\partial B_{2}}\right) p+\left(\frac{\partial \varepsilon}{\partial B_{3}}\right) q\right\} \\
& B=2\left(\frac{\partial \varepsilon}{\partial B_{1}}\right) \frac{K_{13}^{\prime}}{\lambda_{1}^{\mathrm{I}(0)}-\lambda_{3}^{\mathrm{I}(0)}}\left\{-\left(\frac{\partial \varepsilon}{\partial B_{2}}\right) q+\left(\frac{\partial \varepsilon}{\partial B_{3}}\right) p\right\} .
\end{aligned}
$$

The derivatives of the dielectric constant appearing in eqn (31) and (32) can be expressed in terms of more familiar quantities. From the definition of the $\beta$ variables, eqn (11), it follows that 
where

$$
\begin{aligned}
\frac{\partial \varepsilon}{\partial B_{1}} & =\left[k_{\mathrm{B}} T^{2} V / \rho C_{p}\right]^{\frac{1}{2}}\left(\frac{\partial \varepsilon}{\partial T}\right)_{p c_{1} c_{2}} \\
\frac{\partial \varepsilon}{\partial B_{2}} & =\left[k_{\mathrm{B}} T V / \rho \hat{\mu}_{11}\right]^{\frac{1}{2}}\left(\frac{\partial \varepsilon}{\partial c_{1}}\right)_{p T c_{2}} \\
\frac{\partial \varepsilon}{\partial B_{3}} & =\left[k_{\mathrm{B}} T V / \rho\left(\frac{\partial \hat{\mu}_{2}}{\partial c_{2}}\right)_{p T \hat{\mu}_{1}}\right]^{\frac{1}{2}}\left(\frac{\partial \varepsilon}{\partial c_{2}}\right)_{p T \hat{\mu}_{1}}
\end{aligned}
$$

$$
\left(\frac{\partial \varepsilon}{\partial c_{2}}\right)_{p T \hat{\mu}_{1}}=\left(\frac{\partial \varepsilon}{\partial c_{2}}\right)_{p T c_{1}}-\frac{\hat{\mu}_{12}}{\hat{\mu}_{11}}\left(\frac{\partial \varepsilon}{\partial c_{1}}\right)_{p T c_{2}} .
$$

The Stokes and anti-Stokes Brillouin lines are each others mirror image and are given by the sum of a lorentzian and non-lorentzian term

$$
\begin{aligned}
I_{\text {Stokes }}^{\mathrm{B}}(k, \omega)=I_{\text {anti-Stokes }}^{\mathrm{B}}(k,-\omega) & = \\
& \quad \frac{1}{\pi}\left\{Z^{\mathrm{B}} \frac{\Gamma}{\Gamma^{2}+\left(\omega+k c_{0}\right)^{2}}+Y^{\mathrm{B}} \frac{\left(\omega+k c_{0}\right)}{\Gamma^{2}+\left(\omega+k c_{0}\right)^{2}}\right\} .
\end{aligned}
$$

The strength factor of the lorentzian contribution to the Brillouin peaks can be written, using the eigenvector elements given by eqn (28), as

$$
\begin{aligned}
Z^{\mathrm{B}} & =\frac{1}{2}\left(\frac{\partial \varepsilon}{\partial B_{4}}\right)^{2} \\
& =\frac{1}{2}\left(k_{\mathrm{B}} T V / \chi_{\mathrm{s}}\right)\left(\frac{\partial \varepsilon}{\partial p}\right)_{s c_{1} c_{2}}^{2}
\end{aligned}
$$

and the amplitude $Y^{\mathbf{B}}$ of the non-lorentzian part of the Brillouin peaks can be written as

$$
Y^{\mathrm{B}}=\sum_{j=1}^{3} \frac{1}{2}\left(\frac{\partial \varepsilon}{\partial B_{j}}\right)\left(\frac{\partial \varepsilon}{\partial B_{4}}\right) \frac{K_{j 4}}{k c_{0}}-\left(\frac{\partial \varepsilon}{\partial B_{4}}\right)^{2} \frac{K_{44}-K_{55}}{2 k c_{0}}
$$

\section{DISCUSSION}

From eqn (34) it is clear the the Brillouin peaks for a ternary mixture are rather similar to those for one and two component systems. The frequency shift and the strength factor are given by the same expressions as in the case of one and two component systems. The damping factor, eqn (27), consists of four terms, two of which, $\Gamma_{\text {heat conduction }}$ and $\Gamma_{\text {viscosity }}$ have the same form as in the case of one and two component systems. The remaining two terms, $\Gamma_{\text {diffusion }}$ and $\Gamma_{\text {thermal diffusion }}$ are appropriate generalizations of the corresponding terms in a binary mixture to the case where more than one diffusion and thermal diffusion coefficient are present. The number of contributions to the non-lorentzian part of the Brillouin peaks increases with the number of components; however, since the various parts may have opposite signs it is difficult to make general statements about the relative importance of the nonlorentzian part with increasing number of components.

With increasing number of components the Rayleigh peak (eqn (30)) assumes rapidly a more complicated structure. However, the perturbation calculation allows one to obtain rather transparent expressions for the factors determining the shape, eqn (24), and intensity, eqn (31) of the Rayleigh peak. As illustrative examples we now discuss two special cases for which, it turns out, the expressions for the Rayleigh peak take on a rather simple form. 
We first consider the case that one of the species is present in very low concentration, specifically we consider the case that $c_{2}$ tends to zero. From an analysis ${ }^{18}$ of the diffusion equation for this component it follows that, when $c_{2}$ tends to zero, $D_{2}^{T}$ and $D_{21}$ tend to zero proportional to $c_{2}$. Physically it is clear that $D_{22}$ remains finite. From the thermodynamics of dilute solutions ${ }^{19}$ it follows that $\hat{\mu}_{22}$ goes to infinity like $c_{2}^{-1}$ whereas $\hat{\mu}_{11}$ and $\hat{\mu}_{12}$ remain finite. From the above dependence it follows that $K_{13}\left(K_{31}\right)$ and $K_{23}\left(K_{32}\right)$ tend to zero like $c_{2}^{\frac{1}{2}}$. In the limit of $c_{2} \rightarrow 0$ the real roots of $\boldsymbol{K}^{\mathrm{I}}$ are then given by

$$
\begin{aligned}
\lambda_{1,2} & =\frac{1}{2}\left\{\left(D_{\mathrm{T}}+D_{11}\right) \pm\left[\left(D_{\mathrm{T}}-D_{11}\right)^{2}+4\left(D_{1}^{T}\right)^{2} \frac{\hat{\mu}_{11}}{C_{p} T}\right]^{\frac{1}{2}}\right\} k^{2} \\
\lambda_{3} & =D_{22} k^{2}
\end{aligned}
$$

and the corresponding strength factors are

$$
\begin{aligned}
& Z_{1}=\left\{\left(\frac{\partial \varepsilon}{\partial B_{1}}\right) r+\left(\frac{\partial \varepsilon}{\partial B_{2}}\right) s\right\}^{2} \\
& Z_{2}=\left\{-s\left(\frac{\partial \varepsilon}{\partial B_{1}}\right)+\left(\frac{\partial \varepsilon}{\partial B_{2}}\right) r\right\}^{2} \\
& Z_{3}=\left(\frac{\partial \varepsilon}{\partial B_{3}}\right)^{2} .
\end{aligned}
$$

Here

$$
\begin{aligned}
& r=\left[\frac{\lambda_{1}-D_{11} k^{2}}{\lambda_{1}-\lambda_{2}}\right]^{\frac{1}{2}} \\
& s=\operatorname{sign}\left(D_{1}^{\mathrm{T}}\right)\left[\frac{\lambda_{2}-D_{11} k^{2}}{\lambda_{2}-\lambda_{1}}\right]^{\frac{1}{2}} .
\end{aligned}
$$

The eigenvalues $\lambda_{1,2}$ and the corresponding strength factors $Z_{1,2}$ are in fact those encountered in a binary mixture. It should be pointed out, however, that the corresponding normal modes are a linear combination of the variables $\delta s_{\text {red }}$ and $\delta c_{1, \text { red }}$ and not, as in an ordinary binary mixture, a combination of $\delta s_{\text {red }}$ and $\delta c_{1}$. The reason for this is that, as can be seen from the diffusion equation for component 1 , $\delta c_{1}$ is coupled to $\delta c_{2}$ through $D_{12}$ which coefficient (see eqn (8)) does not go to zero as $c_{2}$ tends to zero. On the other hand $\delta c_{1 \text {,red }}$ is coupled to $\delta c_{2}$ only through $D_{21}$ and it is this coupling coefficient that goes to zero when $c_{2}$ tends to zero.

Taking into account that $\hat{\mu}_{22}$ tends to infinity it follows from eqn (33) and (38) that $Z_{3} \ll\left(Z_{2}+Z_{3}\right)$ and thus the lorentzian contribution due to the impurity may not be discernible. However, there are conditions under which it could be observed, for example in the case where the impurity is a macromolecular species such that $D_{22} \ll D_{11}$ and $\left(\partial \varepsilon / \partial c_{2}\right)_{p T c_{1}}^{2} \gg\left(\partial \varepsilon / \partial c_{1}\right)_{p T c_{2}}^{2}$. Then the contribution due to the impurity could appear as a narrow peak with low, but sufficient intensity to be seen above a relatively broad background. Thus one might extract the diffusion coefficient of a macromolecular species in a mixed solvent ${ }^{10}$ in a manner analogous to that currently in use for binary systems. ${ }^{20,21}$

We now consider the case that both components 1 and 2 are present in very low concentrations. From analysis ${ }^{18}$ of the diffusion equations for components 1 and 2 it follows that $D_{1}^{T}$ and $D_{12}$ tend to zero like $c_{1}$ and $D_{2}^{\mathrm{T}}$ and $D_{21}$ tend to zero like $c_{2}$. Again $D_{11}$ and $D_{22}$ remain finite. From the thermodynamics of dilute solutions it follows that $\hat{\mu}_{11}$ and $\hat{\mu}_{22}$ tend to infinity like $c_{1}^{-1}$ and $c_{2}^{-1}$ respectively whereas $\hat{\mu}_{12}$ 
remains finite. From the above behaviour of transport coefficients and thermodynamic derivatives it follows that $K_{12}\left(K_{21}\right)$ goes to zero like $c_{1}^{\frac{1}{2}}$ and $K_{13}\left(K_{31}\right)$ goes to zero like $c_{2}^{\frac{1}{2}}$. The real roots of $\boldsymbol{K}^{1}$ are then given by eqn (40) where the dependence of $K_{22}, K_{23}$ and $K_{33}$, in the limiting case of $c_{1}$ and $c_{2}$ going to zero, has been taken into account.

$$
\left.\begin{array}{l}
\lambda_{1}=D_{\mathrm{T}} k^{2} \\
\lambda_{2}=D_{11} k^{2} \\
\lambda_{3}=D_{22} k^{2} .
\end{array}\right\}
$$

The damping factors obtained for this limiting case correspond to those obtained by Blum (ref. (2), eqn (5.3) and (5.4)). The comparison of the normal modes associated with the $\lambda_{i}$ to those obtained by Blum is rather less clear, certainly the normal mode associated with $\lambda_{1}$ here is $\delta s_{\text {red }}$ not $\delta s$. The corresponding strength factors are given by

$$
Z_{i}=\left(\partial \varepsilon / \partial B_{i}\right)^{2} \quad(i=1,2,3) .
$$

Due to the fact that $\hat{\mu}_{11}$ and $\hat{\mu}_{22}$ go to infinity, $Z_{2,3}$ will, in general, be smaller than $Z_{1}$. However, $D_{11}, D_{22} \ll D_{\mathrm{T}}$ which means that the lorentzian contribution due to the concentration fluctuations may appear as a narrow peak above a broad background. Thus in the limit of a dilute ternary mixture it may be possible to extract the diffusion constants of the dilute species from the spectrum.

This research was supported by a National Research Council of Canada grant and by the Internationale Instituten voor Fysica en Chemie, gesticht door E. Solvay. The assistance of D. L. Carle is also acknowledged.

${ }^{1}$ For a recent review article on laser light scattering see P. A. Fleury and J. P. Boon, Adv. Chem. Phys., 1973, 24, 1.

${ }^{2}$ L. Blum, J. Chem. Phys., 1969, 50, 17.

${ }^{3}$ P. C. Jordan and J. R. Jordan, J. Chem. Phys., 1966, 45, 2492.

${ }^{4}$ H. N. W. Lekkerkerker and W. G. Laidlaw, Phys. Rev. A, 1973, 7, 1332.

${ }^{5}$ P. Martin in Statistical Mechanics of Equilibrium and Non-Equilibrium, ed. J. Meixner (NorthHolland, Amsterdam, 1965), pp. 122-124.

${ }^{6}$ R. D. Mountain and J. M. Deutch, J. Chem. Phys., 1969, 50, 1103.

${ }^{7}$ C. Cohen, J. W. Sutherland and J. M. Deutch, Phys. Chem. Liquids, 1971, 2, 213.

${ }^{8}$ see, for example, M. Dubois and P. Berge, Phys. Rev. Letters, 1971, 26, 121.

${ }^{9}$ W. I. Goldburg and P. N. Pusey, J. Phys. (Paris), 1972, C1, 105.

${ }^{10}$ N. C. Ford, W. Lee and F. E. Karasz, J. Chem. Phys., 1969, 50, 3098.

${ }^{11}$ H. N. W. Lekkerkerker and W. G. Laidlaw, Phys. Rev. A, 1972, 5, 1604.

${ }^{12}$ S. R. de Groot and P. Mazur, Non-Equilibrium Thermodynamics (North-Holland, Amsterdam, 1962).

${ }^{13}$ J. Meixner and H. G. Reik, Thermodynamik der irreversiblen Prozesse in Handbuch der Physik, ed. S. Flügge (Springer, Berlin, 1959), Vol. III, p. 2.

${ }^{14}$ ref. (12), chap. XI, section 2, eqn (61).

15 ref. (12), chap. VII, section 2.

${ }^{16}$ L. D. Landau and E. M. Lifshitz, Statistical Physics (Pergamon, London, 1958), chap. 12.

${ }^{17}$ G. Goertzel and N. Tralli, Some Mathematical Methods of Physics (McGraw-Hill, New York, 1960), chap. 14.

${ }^{18}$ L. D. Landau and E. M. Lifshitz, Fluid Mechanics (Pergamon, London, 1959), chap. 6, section 58.

${ }^{19}$ H. N. W. Lekkerkerker and W. G. Laidlaw, Phys. Chem. Liquids, 1972, 3, 175.

${ }^{20}$ S. B. Dubin, J. H. Lunacek and G. B. Benedek, Proc. Nat. Acad. Sci. U.S.A., 1967, 57, 1164.

${ }^{21}$ N. C. Ford, F. E. Karasz and J. E. M. Owen, Disc. Faraday Soc., 1970, 49, 228. 